\title{
NUEVOS DATOS SOBRE LA DISTRIBUCIÓN DE MAMÍFEROS (MAMMALIA) EN LAS DUNAS DE ERG CHEBBI (MARRUECOS)
}

\author{
T. Ferrández Verdún ${ }^{1, *}$, A. Ruiz Rocamora ${ }^{1}$, P. Perales Pacheco $^{1}$, I. M. Arnaldos Giner ${ }^{1}$, M. Tapia
} Claro $^{1,4}$, J. A. Alguazas Martínez ${ }^{1}$, A. I. Asensio-Pérez ${ }^{3}$, A. F. Carrillo López ${ }^{1}$, Y. da Silva ${ }^{2}$ \& J. Galián ${ }^{3}$

\begin{abstract}
${ }^{1}$ Sociedad de Estudios Biológicos Iberoafricanos (SEBI). Avda. Teodomiro, $301^{\circ} \mathrm{A} 03300$, Orihuela (Alicante). ORCID:
Trinitario Ferrández Verdú: https://orcid.org/0000-0001-6667-8218; Adrián Ruiz Rocamora: https://orcid.org/00000002-2318-7926; Pablo Perales Pacheco: https://orcid.org/0000-0002-5514-7718; Irene María Arnaldos Giner: https:// orcid.org/0000-0002-3543-0663; Manuel Tapia Claro: https://orcid.org/0000-0001-8160-6095; José Antonio Alguazas Martínez: https://orcid.org/0000-0001-5484-8275; Antonio Félix Carrillo López: https://orcid.org/0000-0001-8206-5286
\end{abstract}

2Jarit asociación civil. C/ Buenos Aires, 1046006 Valencia. Yolande Da Silva: https://orcid.org/0000-0002-0399-5838

${ }^{3}$ Departamento de Zoología y Antropología Física. Facultad de Veterinaria. Universidad de Murcia.

Campus de Espinardo 30100, Murcia. Ana Isabel Asensio-Pérez: https://orcid.org/0000-0001-8050-250X; José Galián Albaladejo: https://orcid.org/0000-0002-1415-3767

${ }^{4}$ Gelaya-Ecologistas en Acción Melilla. C/ Colombia, 17 52080, Melilla.

*Autor para correspondencia: Trinitario Ferrández Verdú E-mail: trinofv@gmail.com

\section{RESUMEN}

El progresivo aumento del turismo de aventura en el entorno de las dunas de Erg Chebbi, amenaza, desde hace casi dos décadas, la conservación del único complejo dunar (Erg) de todo Marruecos. Con el propósito de valorar algunos de los efectos de esta actividad sobre la comunidad de mamíferos locales, entre marzo y abril de 2018 se llevó a cabo un muestreo intensivo utilizando 5 métodos de detección distintos. El objeto principal era evaluar la presencia y distribución actual de las especies de mamíferos presentes, teniendo como referencia las citadas en la zona desde 1900 (Aulagnier et al., 2017). De las 16 especies históricamente citadas, 9 han sido detectadas en el presente trabajo, lo que representa un 56.2\%. Resulta de interés la ausencia total de datos sobre 5 de las especies potencialmente más abundantes como son: Gerbillus amoenus (de Winton, 1902), Gerbillus gerbillus Olivier, 1801, Gerbillus tarabuli (Thomas, 1902), Pachyuromis duprasi Lataste, 1880 y Psammomys obesus Cretzschmar, 1828. Algunos datos apuntan a la drástica reducción de los recursos hídricos experimentada en los últimos años, como una de las causas de la aparente reducción de sus poblaciones. Al mismo tiempo, otras especies de requerimiento hídricos menores, tales como: Meriones libycus Lichtenstein, 1823 o Jaculus jaculus Linnaeus, 1758, presentan un aparente buen estado de conservación. Se confirma la presencia de Poecilictis libyca Hemprich \& Ehrenberg, 1833, tras 32 años de ausencia de datos.

Palabras clave: Mamíferos; Erg Chebbi; Sahara; corología; conservación.

\section{ABSTRACT}

\section{New data on the distribution of mammals in the dunes of Erg Chebbi (Morocco)}

The progressive increase of adventure tourism in the area around the dunes of Erg Chebbi has threatened, for almost two decades, the conservation of the only dune complex (Erg) of Morocco. In order to assess some of the effects of this activity on the local mammal communities, an intensive sampling was carried out between March and April 2018, based on 5 different detection methods. The main objective was to evaluate the presence and current distribution of the mammal species cited in the area since 1900. Of the 16 species historically cited, 9 have been detected in the present work, which represents $56.2 \%$. The total absence of data on 5 of the potentially most abundant species such as Gerbillus amoenus (de Winton, 1902), Gerbillus gerbillus Olivier, 1801, Gerbillus tarabuli (Thomas, 1902), Pachyuromis duprasi Lataste, 1880 and Psammomys obesus Cretzschmar, 1828 is remarkable. Some data point to the drastic reduction of water resources experienced in recent years, as one of the causes of the apparent reduction of their populations. At the same time, other species with lower water requirement, such as Meriones libycus Lichtentstein, 1823 or Jaculus jaculus Linnaeus, 1758, have an apparent good state of conservation. The presence of Poecilictis libyca Hemprich \& Ehrenberg, 1833 is confirmed after 32 years without being cited.

Keywords: Mammals; Erg Chebbi; Sahara; carnivores; corology; conservation. 
Cómo citar este artículo/Citation: Ferrández Verdú, T., Ruiz Rocamora, A., Perales Pacheco, P., Arnaldos Giner, I. M., Tapia Claro, M., Alguazas Martínez, J. A., Asensio-Pérez, A. I., Carrillo López, A.F., da Silva, Y. \& Galián, J. 2019. Nuevos datos sobre la distribución de mamíferos (Mammalia) en las dunas de Erg Chebbi (Marruecos). Grael/sia, 75(1): e091. https://doi. org/10.3989/graellsia.2019.v75.218

Copyright: (C) 2019 SAM \& CSIC. This is an open-access article distributed under the terms of the Creative Commons Attribution 4.0 International (CC BY 4.0) License.

\section{Introducción}

Los "mares de arena" o "erg" son sistemas dunares (móviles o fijos), cuya complejidad depende, en buena medida, de la dinámica eólica a la que estén sometidos, pudiendo formarse desde simples ondulaciones eólicas hasta dunas complejas (Wilson, 1972; Lancaster, 1994). De tamaño muy variable, pueden llegar a ocupar superficies que abarcan miles de kilómetros cuadrados (Thomas, 1997), quedando los contornos a menudo formados por superficies planas de arena o mantos de arena (sand sheets). Uno de los elementos de mayor interés natural de estos complejos es su capacidad para acumular, en ocasiones, importantes cantidades de agua subterránea de vital importancia para las comunidades de flora y fauna presentes en la zona.

El complejo dunar de Erg Chebbi, situado en la parte más meridional del extremo oriental de la región de Draa-Tafilalet, constituye un acuífero libre que durante décadas ha abastecido a las poblaciones situadas en su periferia. Las escasas precipitaciones de la región y, por tanto, la recarga reducida que recibe el acuífero, hacen de él un sistema extremadamente delicado que requiere ser gestionado desde el conocimiento científico (García-Rodríguez \& Antón, 2014). Erg Chebbi es reconocido por la UNESCO como parte de la reserva de la Biosfera (Oasis de Taffilalet) y en el Programa de las Naciones Unidas para el Medio Ambiente (UNEP, 2004), la zona es considerada como un área natural de especial protección por su extremada fragilidad.

El presente trabajo, llevado a cabo entre los meses de marzo y abril de 2018, se ha desarrollado en el contexto de una acción más amplia encaminada a conocer el estado actual de la fauna asociada al complejo dunar, que permita establecer criterios objetivos para su conservación integral. En la actualidad, este espacio natural se encuentra muy desprotegido ante la creciente afluencia del turismo de aventura (todoterrenos, motos, quads y buggies) que, desde hace casi dos décadas, se concentra en sus inmediaciones y que, sin ningún tipo de control, está degradando paulatinamente las dunas y su entorno. El área está afectada no sólo por el impacto directo que ocasionan sobre los hábitats el continuo trasiego de personas y vehículos, y la progresiva reducción de los recursos hídricos disponibles que la concentración masiva de personas conlleva; otros impactos como el ruido de los vehículos a motor y determinadas actividades como la caza de fauna para fines turísticos, están provocando el retroceso de la comunidad de mamíferos autóctona, como constatan los habitantes de la zona.

El interés del estudio, por tanto, es disponer de datos que indiquen en qué medida estas actividades turísticas están afectando a la comunidad de mamíferos asociados al complejo dunar. Por ello, el trabajo se centró, por una parte, en detectar la presencia de las especies de este grupo citadas hasta 2016, al objeto de constatar su permanencia en el área; y, por otra, en ampliar los datos sobre su distribución en la zona.

\section{Material y métodos}

\section{Área de ESTUdIO}

Las dunas de Erg Chebbi, que abarcan una superficie de $156 \mathrm{~km}^{2}$, su entorno, así como los núcleos de población más cercanos: Merzouga y Hassilabied pertenecen administrativamente a la provincia de Errachidia (Fig. 1). El interior del complejo dunar se caracteriza por la existencia de grandes dunas piramidales o en estrella, que son las zonas donde se acumulan las mayores cantidades de arena. Con una altitud que oscila entre los $813 \mathrm{~m}$ y los $875 \mathrm{~m}$ de la más elevada, las 14 dunas gigantes existentes en Erg Chebbi pueden elevarse, sobre la superficie media de la arena, más de $100 \mathrm{~m}$. La distancia entre las dunas gigantes varía entre los 1500 y los 2000 m (García-Rodríguez \& Antón, 2014), siendo los vientos predominantes de dirección sureste-este los responsables de su formación (Benallas et al., 2003).

Bajo la arena, actuando como basamento del complejo dunar, aparecen formaciones de esquistos arcillosos de coloración grisácea entre cuyas capas aparecen otras muy finas de areniscas, así como costras ferruginosas del Devónico medio (Fetah et al., 1986).

El clima en Erg Chebbi, de carácter eminentemente desértico presenta, no obstante, algún elemento continental. Su temperatura media anual oscila en torno a $\operatorname{los} 20^{\circ} \mathrm{C}$ con marcados contrastes entre el día y la noche propios de este clima. En verano (julio y agosto) se alcanzan temperaturas máximas de $50^{\circ} \mathrm{C}$, mientras que en diciembre, el mes más frío, la mínima ronda los $5^{\circ} \mathrm{C}$ (García-Rodríguez \& Antón, 2014).

Las precipitaciones anuales están comprendidas entre los $28 \mathrm{~mm}$ y los $129 \mathrm{~mm}$, dependiendo del año hidrológico considerado (Hosni, 2000). Las escasas 


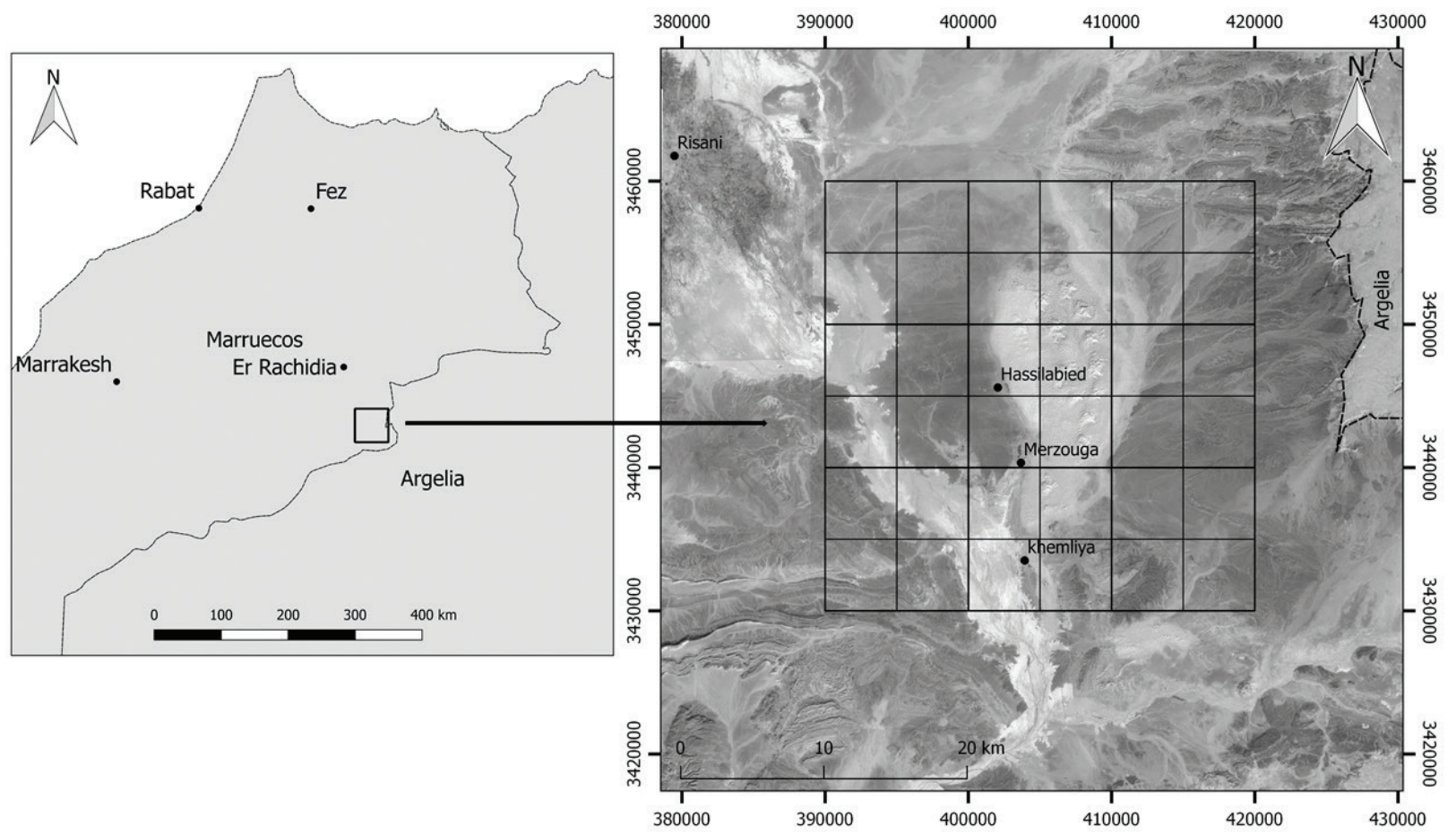

Fig. 1.- Situación general y plano de detalle de la zona de estudio.

Fig. 1.- General situation and detail plan of the study area.

precipitaciones suelen producirse entre los meses de marzo y mayo, aunque hay que destacar las alteraciones pluviométricas puntuales, tormentas ciclónicas, que provocan avenidas relámpago. La climatología dunar aparece condicionada por la presencia de la cordillera del Atlas al norte, que actúa como límite geográfico noroeste de la región. (García-Rodríguez \& Antón, 2014).

Como elemento singular, en la extensa llanura pedregosa que circunda el complejo dunar, se encuentra el lago de Merzouga o "Dayat Tamazguidat": zona endorreica de descarga del acuífero sobre la superficie deprimida de la hamada. Al parecer, y según los propios habitantes de la zona, en condiciones normales, suele tener agua de noviembre a mayo, pudiendo persistir excepcionalmente en el mes agosto (García et al., 2008).

\section{PRINCIPALES FORMACIONES Vegetales}

La zona de estudio perfila las grandes mesetas del Sahara septentrional occidental y se encuentra ya alejada de la influencia del Atlántico y las montañas, con una climatología más árida y marcadamente continental, como se ha comentado, que los territorios más próximos al océano, por lo que hay un empobrecimiento florístico con respecto a éstos. Aun así, Erg Chebbi contiene una considerable riqueza florística con respecto a otras formaciones de vegetación sahariana, ello pese al impacto multisecular de la actividad humana.
Por lo general, el árbol más abundante en la zona es, junto con el taray (Tamarix aphylla), la acacia (Acacia tortilis subsp. raddiana), aunque siempre aparecen de modo más o menos puntual y disperso y suelen hacerlo en bajas densidades. Además de estas especies arbóreas, aparecen las formaciones que se relacionan a continuación:

- Formaciones de Tetraena gaetula. Se trata, a menudo, de comunidades vegetales monoespecíficas que pueden ocupar extensiones de varios cientos de metros cuadrados. En ocasiones, aparecen en asociación con Stipagrostis pungens y Panicum turgidum. Se distribuyen por las partes más bajas de las dunas (pies de duna).

- Formaciones de Stipagrostis pungens. Igual que en el caso anterior, son frecuentes sus formaciones monoespecíficas de extensión variable, entre cientos y miles de metros cuadrados. También se presentan en las áreas externas del complejo dunar.

- Bosquetes dispersos de Tamarix aphylla. Son formaciones monoespecíficas. Pueden estar formados por unas decenas de ejemplares dispersos en una superficie reducida u ocupar una extensión mucho más amplia de cientos de ejemplares, aunque siempre en densidades muy bajas. En ocasiones, ocupan el estrato inferior formaciones laxas de Stipagrostis pungens. Se distribuyen principalmente por el extremo suroriental del sistema dunar. Este taray suele ser un excelente fijador de las dunas y aparece en zonas donde el sustrato basal del suelo es impermeable.

- Palmerales cultivados. De pequeñas dimensiones, los dos existentes, están situados entre el sistema dunar y 
los núcleos de población más cercanos: Merzouga y Hassilabied. Formados por Phoenix dactylifera, ubicadas en los márgenes de los bancales o tablas cultivadas en las que crecen hortalizas y algunos cítricos.

- Dunas. Forman la mayor parte del complejo dunar. Ausente prácticamente en su totalidad de cobertura vegetal, puede albergar ejemplares aislados de especies como: Phoenix dactylifera, Acacia tortilis subsp. raddiana y Calotropis procera, entre otras.

- Hamada o Reg. Extensas planicies predunares, yermas y pedregosas, con ejemplares muy dispersos de Acacia tortilis subsp. raddiana y palmeras (Phoenix dactylifera). Envuelve todas las dunas extendiéndose por amplias superficies de decenas de kilómetros cuadrados.

- Lago de Merzouga. Zona húmeda de origen endorreico, donde proliferan las formaciones de Tamarix amplexicaulis. En la actualidad, la sobreexplotación de los recursos hídricos que sufre la zona ha reducido notablemente la superficie del lago, lo que ha provocado un aumento de la vegetación asociada a la zona húmeda.

\section{REALIZACIÓN DEL CATÁLOGO DE MAMÍFEROS}

La confección del catálogo se llevó a cabo mediante los siguientes recursos metodológicos:

- Revisión bibliográfica de las especies citadas desde 1900 a la actualidad. Para ello se han empleado las referencias de Aulagnier et al. (2017), reseñadas a una escala de cuadrícula de 25 x $25 \mathrm{~km}$.

- Fototrampeo. Se instalaron 23 estaciones de fototrampeo. Las cámaras-trampa permanecieron activas 24 horas/día entre 3 y 5 días en modo video de $15 \mathrm{~s}$. Se cebaron con sardinas en aceite y orina de león como atrayente oloroso.

- Entrevistas. Se realizaron un total de 11 entrevistas, siempre a residentes nativos (agricultores, pastores o habitantes de la zona en general), sobre las especies actuales. Se hacía hincapié en que hubieran sido vistas no hace más de un año y a menos de $10 \mathrm{~km}$ del área de estudio. Para ello se disponía de fotografías a color de las especies potencialmente presentes en las dunas y su entorno.

- Transectos.

- Diurnos. A partir de recorrido a pie por un mínimo de dos personas se llevaron a cabo un total de 11 transectos diurnos. La primera persona se desplazaba en primer lugar y estaba encargada de la detección e identificación de la fauna avistada. Cincuenta o 100 metros detrás, el resto de participantes, se ocupaban de detectar, identificar y fotografiar las huellas y recoger muestras de heces para su análisis. La distancia de los transectos fue variable (entre $500 \mathrm{~m}$ y $2000 \mathrm{~m}$ ), dependiendo de las características e interés del lugar muestreado. También se realizaron un total de 10 transectos diurnos en vehículo con las características que se detallan en el apartado siguiente.

- Nocturnos. A partir de recorridos en coche a una velocidad constante de entre 20 y $30 \mathrm{~km} / \mathrm{h}$, se llevaron a cabo un total de 4 transectos nocturnos. Fueron realizados por un mínimo de tres personas: dos avistadores y un conductor. La distancia de los transectos fue variable (entre $2.5 \mathrm{~km}$ y $14 \mathrm{~km}$ ) dependiendo de las características e interés del lugar muestreado. Nunca se llevaron a cabo en las mismas zonas en las que se realizaron los transectos diurnos.

- Localizaciones fuera de muestreo. Durante los desplazamiento a pie o en coche se anotaron y geolocalizaron los avistamientos, rastros o huellas encontrados incidentalmente, resultando un total de 16.

- $\quad$ Esperas. Se llevó a cabo 1 espera. Se eligió un lugar elevado de alta visibilidad para el avistamiento de la fauna y fue llevado a cabo por 2 equipos de 3 personas. El tiempo de espera fue de 2 horas desde el amanecer.

- Jaulas-trampa. Por motivos ajenos a nuestro interés solo se colocaron 2 jaulas-trampa (1 noche) durante el periodo de muestreo; en ninguna de ambas se obtuvo resultados positivos. Las jaulas, modelo Sherman, fueron colocadas junto al lago de Merzouga o "Dayat Tamazguidat" y se cebaron con barritas energéticas a base de frutos secos y miel.

Para la identificación taxonómica de las muestras de heces de carnívoros se procedió a la extracción del ADN de todas las muestras mediante el kit comercial Favor Prep Stool DNA Isolation Mini Kit, siguiendo las instrucciones establecidas por el fabricante. Las muestra conservadas en propilenglicol, fueron lavadas previamente con etanol absoluto y secadas en la estufa antes de proceder al protocolo de extracción. La amplificación de ADN por PCR se llevó a cabo utilizando las condiciones y los primers universales, ThrL y DLH, descritos por Vilà et al. (1999) y Leonard et al. (2002), que permiten obtener secuencias de la región control del ADN mitocondrial y que permite la identificación de lobos y perros. Las muestras se secuenciaron en Macrogen (Amsterdam, Países Bajos) usando un ABI Prism 3730XL. Las secuencias fueron alineadas en GENIOUS 7.1.7 (Biomatters) y comparadas con otras secuencias de la base de datos GenBank, del National Center for Biotechnology Information (NCBI) mediante la herramienta BLAST (Basic Local Alignment Search Tool) que permite localizar secuencias homólogas que han sido previamente caracterizadas y calcular la significación de los resultados. Solo de 3 muestras se obtuvieron resultados fiables, en concreto las publicadas en la Tabla 3 .

La frecuencia relativa (FR) de cada especie respecto al resto se calculó mediante el cociente entre el número de muestreos con imágenes para dicha especie y el número total de estaciones de fototrampeo instaladas (Moruzzi et al., 2002). La FR no ha sido calculada para las especies que no fueron identificadas a través de fototrampeo.

Entre las especies que sólo han sido detectadas a partir de las entrevistas, se ha incluido, exclusivamente, la única que aparece en el $100 \%$ de las realizadas: Mus spretus Lataste, 1883. Canis anthus F. Cuvier, 1820, también identificado en el 100\% de las entrevistas, no ha quedado incluida dado que, pese 
a tratarse de una especie históricamente citada en el área entre 1951 y 1980 (Aulagnier \& Thévenot, 1986), en ningún caso los entrevistados la ubicaron en la zona de estudio, sino en el entorno de un radio de más de $50 \mathrm{~km}$.

Respecto a las especies de la familia Gerbillidae, no fue posible obtener datos de entrevistas, dado que los entrevistados no diferenciaban entre especies, a excepción de Jaculus jaculus Linnaeus, 1758, para la que sí fue posible la identificación específica a través de imágenes.

\section{Resultados}

En la Tabla 1 se relacionan las especies citadas en la zona desde 1900 según Aulagnier et al. (2017).

\section{LISTADO DE ESPECIES IDENTIFICADAS}

\section{Orden Eulipotyphla}

Familia Erinaceidae

Paraechinus aethiopicus Ehrenberg, 1833 (Erizo del desierto). Detectado mediante fototrampeo, entrevistas rastros y huellas (Tabla 2). Especie abundante en la zona. Ocupa tanto las dunas $(42 \%$ de los contactos) como las áreas predunares periféricas (58\% de los contactos) $(\mathrm{n}=19)$, desde los bosquetes de taray (Tamarix aphilla) hasta la hamada (llanura desértica pedregosa). En Marruecos se distribuye por el extremo meridional y ha sido localizada en 10 cuadriculas del área de estudio (Fig. 2). Estatus UICN: DD.

\section{Orden Chiroptera}

Familia Rhinopomatidae

Asellia tridens Geoffroy, 1813 (Murciélago tridente). Se detectó una colonia por avistamiento directo formada por algunas decenas de ejemplares y otras localizaciones mediante entrevistas (Tabla 2). Siempre en la zona predunar $(n=3$, Fig. 3$)$, la colonia detectada se encontraba refugiada bajo grandes ejemplares de Tetraena gaetula (comportamiento del que no tenemos constancia que haya sido citado anteriormente). $\mathrm{Su}$ distribución nacional abarca la parte meridional del extremo oriental del país. Estatus UICN: LC.

\section{Orden Carnivora}

Familia Canidae

Vulpes vulpes Linnaeus, 1758 (Zorro rojo). Ha sido detectado mediante fototrampeo, transecto nocturno, entrevistas y rastros (análisis genético de heces) (Tablas 2 y 3). Aunque no es muy escaso, su población se considera más escasa que la de $V$. zerda Zimmermann, 1780, tanto a tenor de los datos obtenidos como por la opinión de los entrevistados. Detectados siempre en las zonas predunares $(n=12$, Fig. 4), no parece solapar en exceso sus zonas de campeo con V. zerda. En Marruecos ocupa, casi exclusivamente, la mitad oriental del país. Estatus UICN: LC.

Vulpes zerda Zimmermann, 1780 (Fenek). Identificado a partir de fototrampeo, foqueo nocturno, entrevistas y rastros y huellas (Tabla 2). Su población en Erg Chebbi parece mantener un razonablemente bueno estado de conservación. No obstante, la existencia de un ejemplar con una pata amputada hace sospechar

Tabla 1.- Especies citadas desde 1900 en Erg Chebbi y su entorno a partir de Aulagnier et al. (2017). En gris aparecen las especies detectadas en el estudio.

Table 1.- Species cited since 1900 in Erg Chebbi and its surroundings from Aulagnier et al. (2017). In gray, the species detected in the study appear.

\begin{tabular}{llc}
\hline N. Científico & \multicolumn{1}{c}{ N. Vulgar } & Periodos de la cita \\
\hline Paraechinus aethiopicus & Erizo del desierto & 2001-2016 \\
Asellia tridens & Murciélago tridente & $2001-2016$ \\
\hline Pipistrellus kuhlii & Murciélgo de borde claro & $2001-2016$ \\
Canis anthus & Lobo moruno & $1951-1980$ \\
Vupes vulpes & Zorro rojo & $2001-2016$ \\
Vulpes zerda & Fenek & $2001-2016$ \\
Poecilictis libyca & Zorrilla del desierto & $1981-2000$ \\
Lepus mediterraneus & Liebre magrebi & $2001-2016$ \\
Jaculus jaculus & Jerbo de Egipto & $2001-2016$ \\
\hline Gerbillus amoenus & Jerbillo enano de Egipto & $2001-2016$ \\
Gerbillus gerbillus & Pequeño jerbillo de Egipto & $2001-2016$ \\
Gerbillus tarabuli & Jerbillo de Libia & $2001-2016$ \\
Pachyuromys duprasi & Rata del desierto de cola crasa & $\mathbf{2 0 0 1 - 2 0 1 6}$ \\
\hline Meriones libycus & Rata de cola roja & $2001-2016$ \\
\hline Psammomys obesus & Jird gordo & $2001-2016$ \\
\hline Mus spretus & Ratón moruno & $2001-2016$ \\
\hline
\end{tabular}


Tabla 2.- Estadística de detección de las especies y frecuencia relativa (FR). Avistamiento (avis); huellas y rastros $(\mathrm{h} / \mathrm{r})$.

Table 2.- Statistics of species detection and relative frequency (FR). Sighting (avis); track and trail (h/r)

\begin{tabular}{lcccc}
\hline Especie & $\mathbf{N}^{\circ}$ de Fototrampeo & FR & Transectos Diurnos/Nocturnos & Entrevistas \\
\hline Paraechinus aethiopicus & 6 & $26 \%$ & $2(\mathrm{~h} / \mathrm{r})$ & $11(100 \%)$ \\
Asellia tridens & - & & $1(\mathrm{avis})$ & $2(18 \%)$ \\
Vulpes vulpes & 2 & $8 \%$ & $3(\mathrm{avis}) ; 3(\mathrm{~h} / \mathrm{r})$ & $4(36 \%)$ \\
Vulpes zerda & 4 & $17 \%$ & $1(\mathrm{avis}) ; 3(\mathrm{~h} / \mathrm{r})$ & $11(100 \%)$ \\
Poecilictis libyca & 1 & $4 \%$ & - & $9(82 \%)$ \\
Lepus mediterraneus & - & & $2(\mathrm{~h} / \mathrm{r})$ & $9(82 \%)$ \\
Jaculus jaculus & 10 & $43 \%$ & $5(\mathrm{avis}) ; 8(\mathrm{~h} / \mathrm{r})$ & $11(100 \%)$ \\
Meriones libycus & 7 & $30 \%$ & $3(\mathrm{~h} / \mathrm{r})$ & - \\
Mus spretus & - & & - & $11(100 \%)$ \\
\hline
\end{tabular}

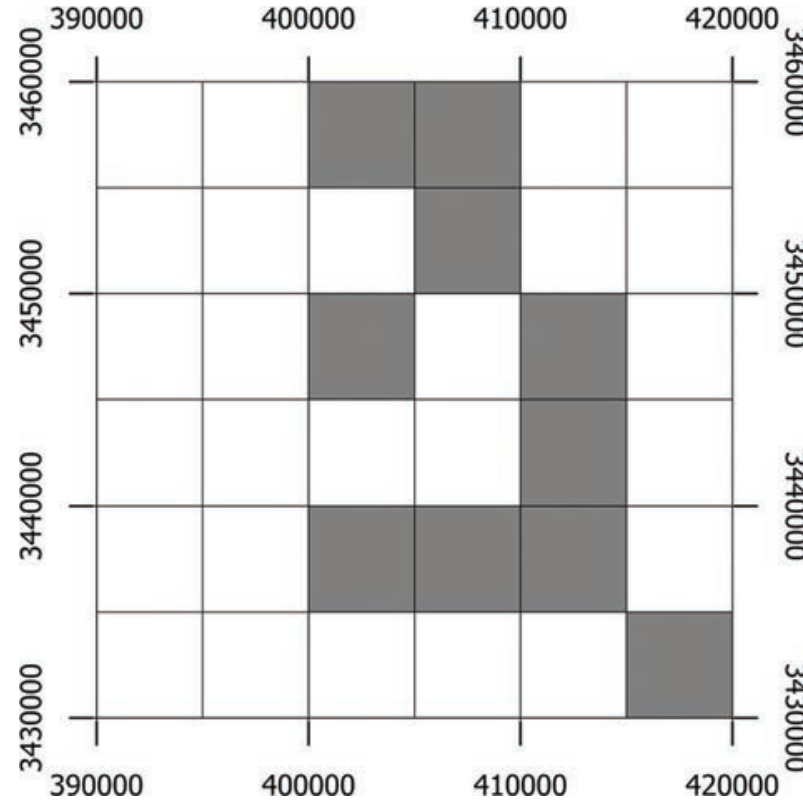

Fig. 2.- Área de estudio y cuadrículas en la que ha sido detectada la especie Paraechinus aethiopicus.

Fig. 2.- Paraechinus aethiopicus. Study area and grids in which the species has been detected.

de la existencia de trampas para su captura o muerte. Localizado mayoritariamente en el interior de las dunas (58\% de los contactos) $(\mathrm{n}=19$, Fig. 5), siempre aparece asociado a espacios con vegetación (bosquetes de taray, herbazales de Tetraena gaetula o formaciones de Panicum turgidum). En Marruecos ocupa principalmente amplias áreas del extremo suroccidental. Estatus UICN: LC.

Familia Mustelidae

Poecilictis libyca Hemprich y Ehrenberg, 1833 (Zorrilla del desierto). Identificada por fototrampeo y a través de entrevistas (Tabla 2). Ha sido detectada en el interior de las dunas $(60 \%$ de los

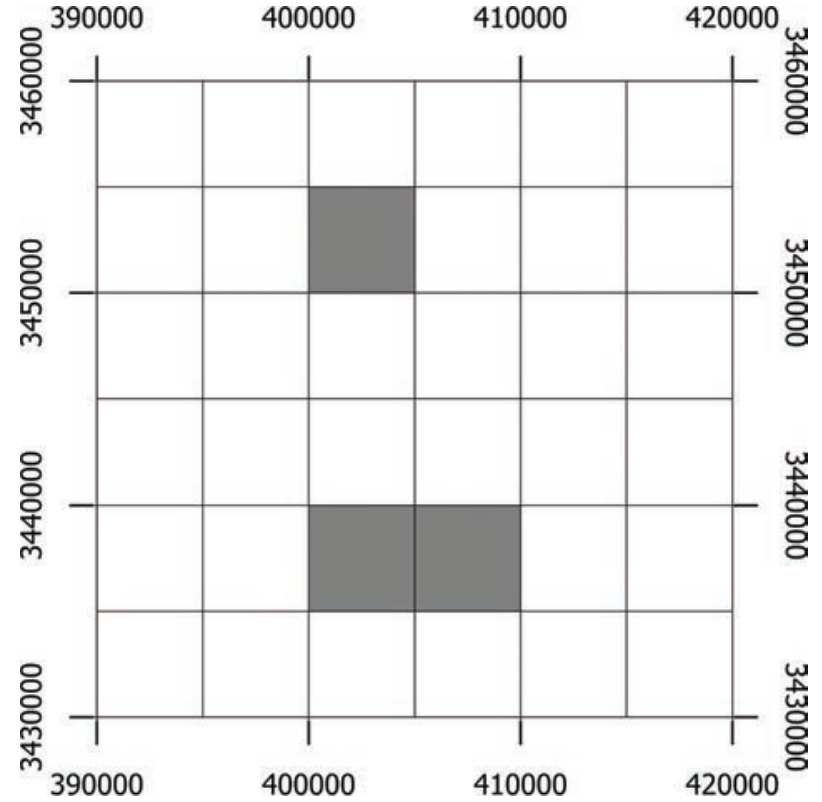

Fig. 3.- Área de estudio y cuadrículas en la que ha sido detectada la especie Asellia tridens.

Fig. 3.- Asellia tridens. Study area and grids in which the species has been detected.

contactos), aunque también ocupa los espacios externos predunares $(40 \%$ de los contactos $)(n=10$, Fig. 6). Desde hace 32 años no había sido citada en la zona (Aulagnier y Thévenot, 1986). Su distribución actual en Marruecos ocupa principalmente el extremo sur. Estatus UICN: LC.

\section{Orden Lagomorpha}

Familia Leporidae

Lepus mediterraneus Wagner, 1841 (Liebre magrebí). Identificada a través de rastros y huellas y mediante entrevistas (Tabla 2). A tenor de los resultados y por conversaciones con los residentes en la zona, se trata de una especie escasa. Localizada tanto en las propias 
dunas (27\% de los contactos) como en las áreas periféricas predunares ( $73 \%$ de los contactos) $(\mathrm{n}=11)$, ocupa tanto lo pequeños bosquetes de taray, como las extensos espacios de hamada pedregosa (Fig. 7). En Marruecos aparece prácticamente en todo el país a excepción de las zonas desérticas interiores del extremo sur. Estatus UICN: LC.

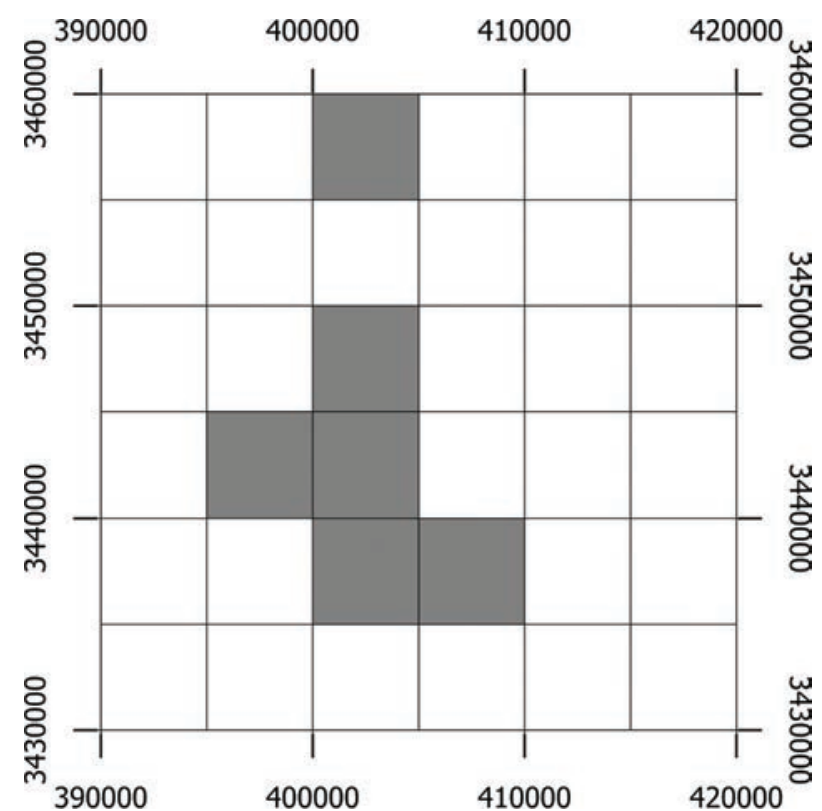

Fig. 4.- Área de estudio y cuadrículas en la que ha sido detectada la especie Vulpes vulpes.

Fig. 4.- Vulpes vulpes. Study area and grids in which the species has been detected.

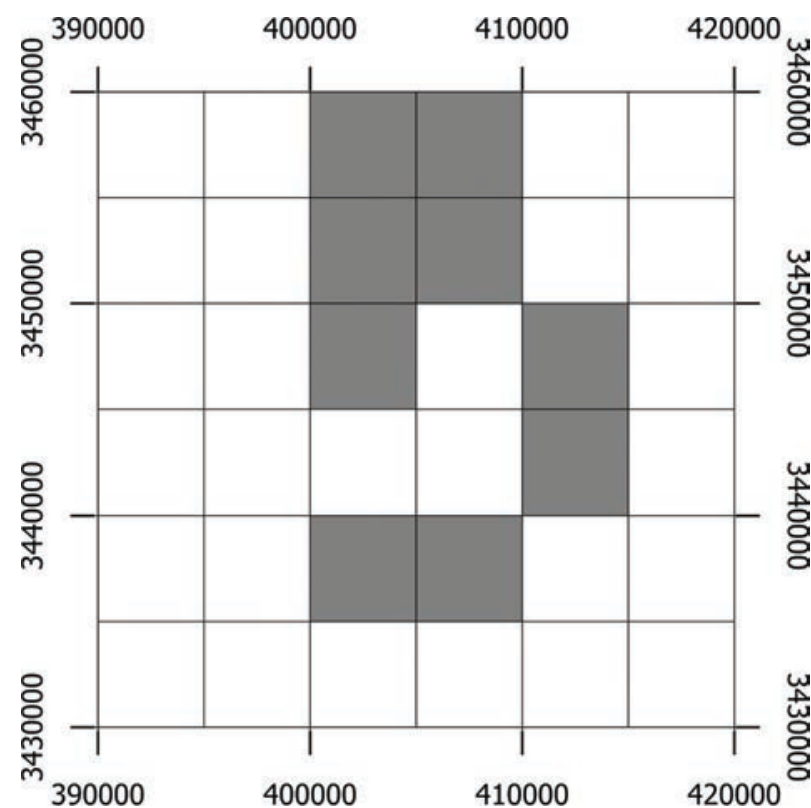

Fig. 5.- Área de estudio y cuadrículas en la que ha sido detectada la especie Vulpes zerda.

Fig. 5.- Vulpes zerda. Study area and grids in which the species has been detected.
Orden Rodentia

Familia Dipodidae

Jaculus jaculus Linnaeus, 1758 (Jerbo de Egipto). Esta especie de Jerbo ha sido detectada a partir de fototrampeos, transectos nocturnos, entrevistas, huellas y rastros (Tabla 2). Es una especie muy abundante, tanto en las zonas dunares ( $24 \%$ de los contactos) como en las

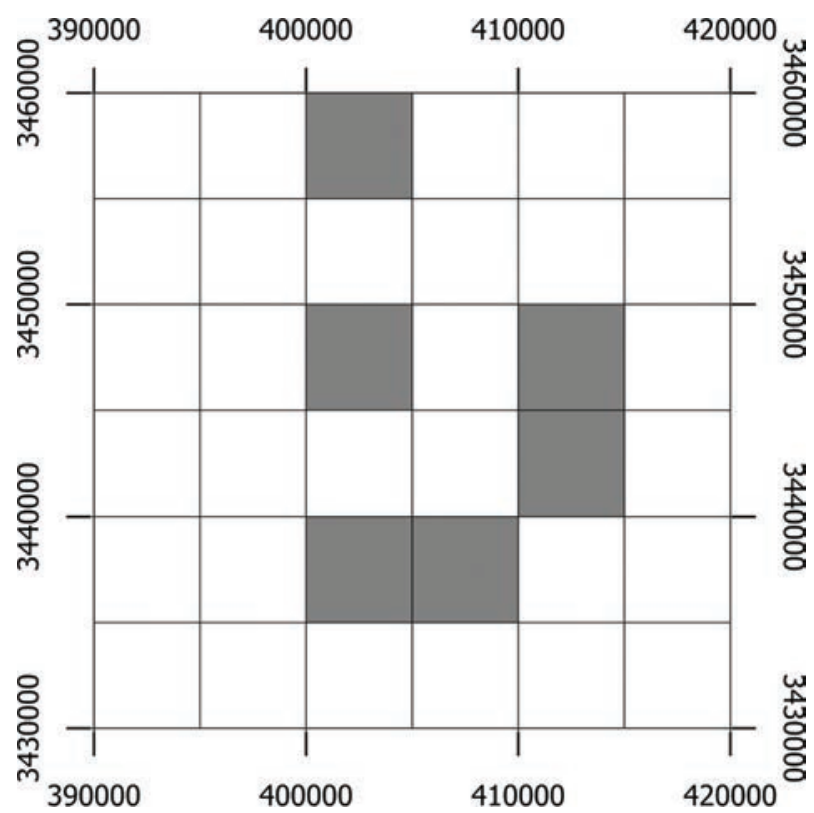

Fig. 6.- Área de estudio y cuadrículas en la que ha sido detectada la especie Poecilictis libyca.

Fig. 6.- Poecilictis libyca. Study area and grids in which the species has been detected.

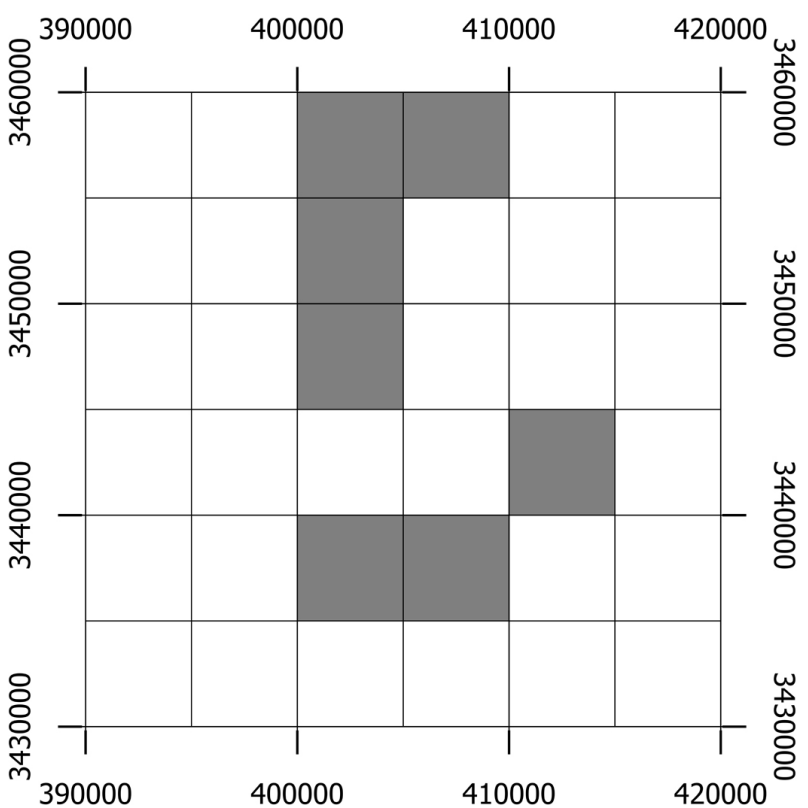

Fig. 7.- Área de estudio y cuadrículas en la que ha sido detectada la especie Lepus mediterraneus.

Fig. 7.- Lepus mediterraneus. Study area and grids in which the species has been detected. 
predunares periferias $(76 \%$ de los contactos $)(n=34)$. Localizado tanto en formaciones boscosas abiertas de taray y pequeños palmerales, como en estepas de gramíneas y hamadas pedregosas (Fig. 8). Estatus UICN: LC.

\section{Familia Gerbillinae}

Meriones libycus Lichtenstein, 1823 (Rata de cola roja) Identificados a través de fototrampeo y rastros (Tabla 2). Se trata del único Gebillinae detectado. Abundantes, ocupa tanto las zonas dunares ( $40 \%$ de los contactos) como las áreas de hamada predunares $(60 \%$ de los contactos) ( $n=10$, Fig. 9). Generalmente localizados en el interior de formaciones vegetales más o menos laxas de taray, acacias, palmeras o gramíneas. Estatus UICN: LC.

\section{Familia Muridae}

Mus spretus Lataste, 1883 (Ratón moruno). Identificado exclusivamente a través de entrevistas (Tabla 2). Según los informantes se trata de una especie común, sobre todo en las inmediaciones de los poblados (Fig. 10). Estatus UICN: LC.

De las 6 especies detectadas mediante fototampeo: 3 carnívoros (Vulpes vulpes, Vulpes zerda y Poecilictis libyca) y 3 micromamíferos (Paraechinus aethiopicus, Meriones libycus y Jaculus jaculus), son estas últimas, como cabría esperar, las que presentan una mayor frecuencia relativa con el $26 \%, 30 \%$ y $43 \%$, respectivamente (Tabla 2). Se trata también de las 3 especies que presentan una mayor distribución en el área, ocupando el $28 \%, 22 \%$ y $39 \%$, respectivamente de las cuadrículas totales muestreadas.

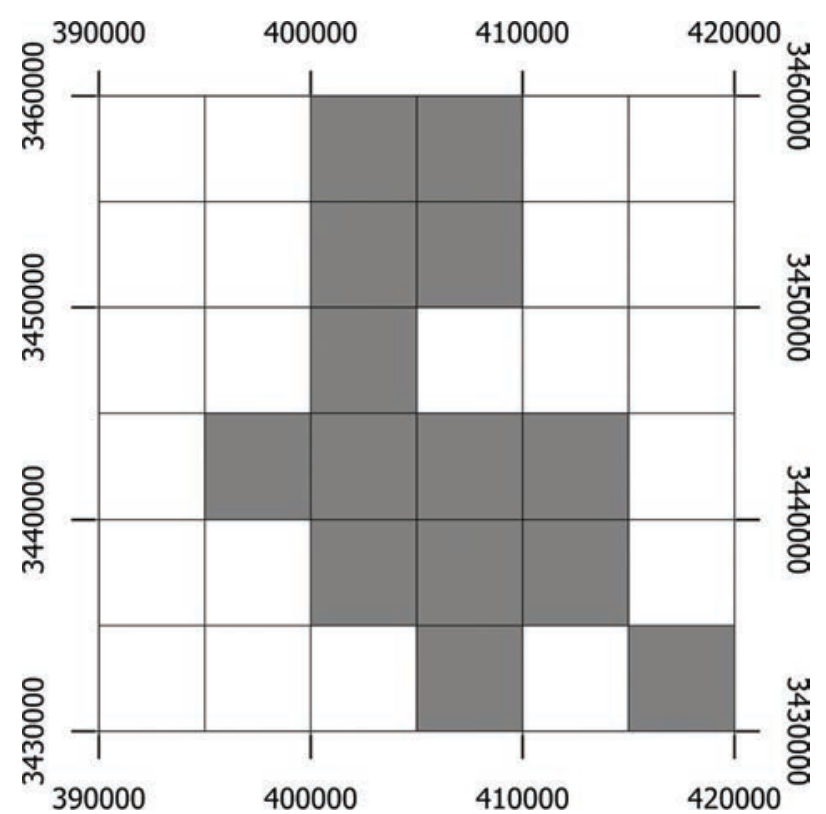

Fig. 8.- Área de estudio y cuadrículas en la que ha sido detectada la especie Jaculus jaculus.

Fig. 8.- Jaculus jaculus. Study area and grids in which the species has been detected.
Todas las especies detectadas han mantenido un uso de los hábitats definidos (dunar y predunar) compartida: en ningún caso se ha detectado un uso exclusivo de alguno de los hábitats por parte de una especie. No obstante, sí se ha observado una discriminación positiva de los espacios con cobertura vegetal (67\% de los

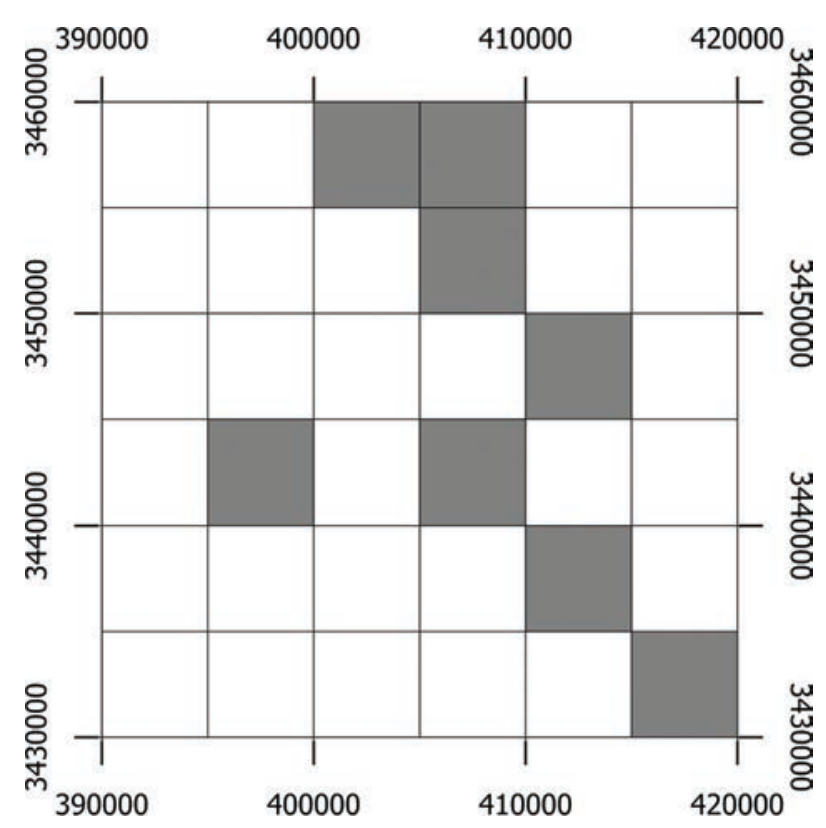

Fig. 9.- Área de estudio y cuadrículas en la que ha sido detectada la especie Meriones libycus.

Fig. 9.- Meriones libycus. Study area and grids in which the species has been detected.

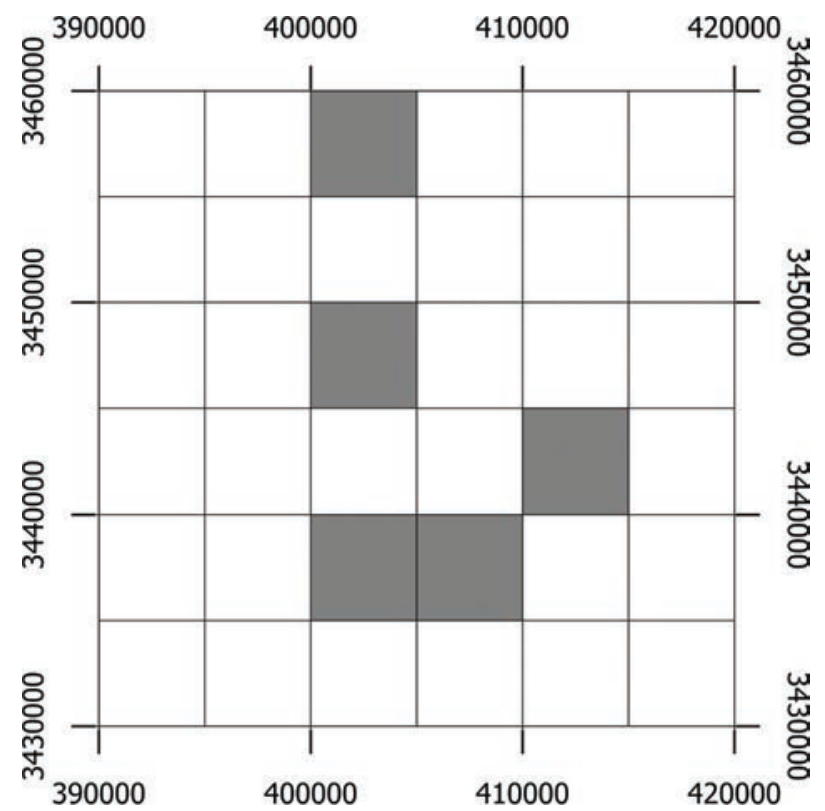

Fig. 10.- Área de estudio y cuadrículas en la que ha sido detectada la especie Mus spretus.

Fig. 10.- Mus spretus. Study area and grids in which the species has been detected. 
Tabla 3.- Resultados de los análisis de secuencias de ADN realizados en las muestras de heces colectadas.

Table 3.- Results of DNA sequence analysis performed on stool samples collected.

\begin{tabular}{lccc}
\hline Sequence (HQ) & BLAST-ID (E-Value) & Grade & GenBank_ID \\
\hline 1 A (29.3-35.7\%) & Vulpes vulpes (0) & $73.6 \%$ & KJ598008 \\
& Vulpes vulpes (0) & $73.1 \%$ & KJ598015 \\
& Vulpes vulpes (0) & $72.8 \%$ & KJ598017 \\
1 B (83.2\%) & Vulpes vulpes (0) & $96.8 \%$ & AB292760 \\
& Vulpes vulpes (0) & $96.8 \%$ & AB292758 \\
& Vulpes vulpes (0) & $96.7 \%$ & AB292759 \\
2 B (80.3\%) & Vulpes vulpes (0) & $96.7 \%$ & AB292760 \\
& Vulpes vulpes (0) & $96.7 \%$ & AB292758 \\
& Vulpes vulpes (0) & $96.6 \%$ & AB292759 \\
\hline
\end{tabular}

contactos) frente a los que no la presentaban (33\%), pese a que ésta nunca ha superado el $25 \%$ de cobertura.

A partir de los análisis genéticos realizados en las muestras de heces colectadas, se han obtenido los resultados que se muestran en la Tabla 3.

\section{Discusión}

De las 16 especies de mamíferos citadas desde 1900 en las dunas de Erg Chebbi (Aulagnier et al., 2017), 9 han sido detectadas a nivel de especie por, al menos, uno de los métodos de muestreo empleados, lo que representa el $56.2 \%$. De las 7 especies no encontradas, 5 pertenecen a la familia Gerbillinae: Gerbillus amoenus (de Winton, 1902), Gerbillus gerbillus Olivier, 1801, Gerbillus tarabuli (Thomas, 1902), Pachyuromis duprasi Lataste, 1880, y Psammomys obesus Cretzschmar, 1828. En general, se trata de especies con poblaciones relativamente abundantes allí donde aparecen, tal es el caso de Gerbillus amoenus con densidades de $83 \mathrm{ind} / \mathrm{ha}$ (Berrada, 1986) o Psammomys obesus con valores de 50-100 ind/ha (Petter, 1987), por lo que la ausencia total de datos, junto al hecho de haber detectado buenas poblaciones de otras como M. libycus o J. jaculus, potencialmente mucho menos abundantes, como es el caso de M. libycus con densidades de $0.05 \mathrm{ind} / \mathrm{ha}$ (Petter, 1987) con las que comparten hábitat y costumbres, tal vez nos esté indicando la existencia de ciertos problemas que podrían estar afectando al mantenimiento de sus poblaciones. Es decir, si especies citadas en la zona en los últimos 15 años como las que nos ocupan (Aulagnier et al., 2017), con una habitualmente alta densidad de población en sus áreas de distribución, no han sido detectadas y sí lo han sido otras con una significativamente menor densidad, es razonable sospechar que las primeras estén o hayan estado sometidas, en los últimos años, a algún tipo de presión ambiental de resultados negativos.
La disminución de los niveles freáticos y de las aguas superficiales asociadas, que desde hace unos años tienen lugar debido a la sobreexplotación de los recursos hídricos, como en el lago de Merzouga (García et al., 2008), unido a la desaparición de algunas de las especies vegetales asociadas a estos ambientes lacustres y su consiguiente producción de semillas, parece estar en la base de la escasa diversidad de gerbillos observada cuya disponibilidad simultánea de agua y energía es el principal factor que explica la variación de su riqueza específica (Petter et al., 1984; Real et al., 2003). En este sentido, la abundancia de gatos cimarrones detectada (datos no mostrados), tanto en las áreas dunares como predunares, también podría ser un factor de presión sobre las poblaciones de estos micromamíferos.

\section{Conclusiones}

De las cuatro especies de carnívoros citadas desde 1900 (Tabla 1), tres han sido detectadas y la cuarta, Canis anthus, aunque históricamente ocupó la zona y en la actualidad no parece estar presente, es identificada y reconocida por los entrevistados como especie que ocupa las áreas de montaña en las zonas periféricas en un radio aproximado de $50 \mathrm{~km}$.

La ausencia total de datos sobre 5 de las especies potencialmente más abundantes como son: Gerbillus amoenus, G. gerbillus, G. tarabuli, Pachyuromis duprasi y Psammomys obesus, hace temer que se haya producido una drástica reducción de sus poblaciones, cuando no su extinción. Al mismo tiempo, especies de requerimiento ecológicos similares, tales como: Meriones libycus o Jaculus jaculus, presentan un aparente buen estado de conservación, circunstancias que podrían estar relacionadas con su menor dependencia del agua (Petter, 1961), un recurso cada vez más limitante.

Así pues, al tiempo que por el efecto directo de la actividad turística y la degradación asociada al hábitat dunar, que estimamos afecta de manera directa al estado de los hábitats y sus poblaciones, la reducción de la biodiversidad detectada podría estar asociada a la reducción de los recursos hídricos que esta actividad está causando en el acuífero de Erg Chebbi.

Serían necesarios nuevos trabajos que permitieran establecer con precisión el estado de las poblaciones de las especies presentes, así como en qué medida ambas circunstancias -reducción de especies y de recursos hídricos- están relacionadas, al tiempo que se ven afectadas de forma directa por las actividades turísticas que se desarrollan en su medio.

\section{Agradecimientos}

A Elisa Arnaldos Giner y Josh Keane por la revisión del texto en inglés. A Raimundo Real y Ramón C. Soriguer por la revisión crítica del manuscrito. A todas las personas anónimas que, con su amabilidad y conocimientos sobre la fauna local, han contribuido de forma desinteresada a la realización de este trabajo. 


\section{Referencias}

Aulagnier, S., Cuzin, F. \& Thévenot, M. 2017. Mammifères sauvages du Maroc: peuplement, répartition, écologie. Société française pour l'étude et la protection des mammifères. Paris. 339 pp.

Aulagnier, S. \& Thevenot, M. 1986. Catalogue des mammifères sauvages du Maroc. Ministère de l'education nationale, Université Mohammed V, Institut scientifique.

Benallas, M., Alen, E. M., Rognon, P., Desjardins, R., Hilari, A. \& Khardi, A. 2003. Eolian dynamic and palm grove encroachament in the sand dunes of Tafilatet. Sciences et Changement Planétaires. Sécheresse, 14(2): 77-83.

Berrada, M. 1986. Approche expérimentale d'une méthode d'échantillonnage sur bandes lineaires pour le suivi des population des rongeurs: Gerbillus nanus, Meriones shawii et Psammomys obesus. Mémoire d'Ingénieur d'État en Phytopatologie. Institut Agronómique et Vétérinaire Hassan II. Agadir. 97 pp.

Fetah, S. E., Bensaïd, M. \& Dahmai, M. 1986. Carte Géologique du Maroc. Tafilalt-Taouz, Echelle 1/200.000. Service Géologique du Maroc. Rabat.

García, M., Moya-Palomares, M ${ }^{\mathrm{a}}$. E., De Pablo, M. A., Vicente, R. \& Acaso, E. 2008. Nuevas aportaciones sobre el funcionamiento hidrogeológico del acuífero de Erg Chebbi en el entorno de Hassilabied (Marruecos). M+A. Revista Electrónic@,de Medio Ambiente, 5: 41-57. Disponible en: https://www.researchgate.net/ publication/258516017 Nuevas aportaciones sobre el_funcionamiento_hidrogeologico_del_acuifero_de Erg_Chebbi_en_el_entorno_de_Hassilabied_Marruecos [consultado: $4.0 \overline{4} .2019]$

García-Rodríguez, M. \& Antón, L. 2014. Aplicación de SIG para la caracterización hidrogeológica de sistemas dunares (Erg Chebbi, Marruecos). Estudios Geográficos, 75(277): 575-596.

Hosni, E. 2000. Strategy for sustainable tourism development in the Sahara. UNESCO. Paris. $71 \mathrm{pp.}$

Lancaster, N. 1994. Dune morphology and dynamics. In: A. D. Abrahams \& A. J. Parson (eds.). Geomorphology of Desert Environments. Chapman and Hall. London: 474-505.

Leonard, J. A., Wayne, R. K., Wheeler, J., Valadez, R., Guillén, S. \& Vila, C. 2002. Ancient DNA evidence for Old World origin of New World dogs. Science, 298(5598): 1613-1616. https://doi.org/10.1126/science.1076980

Moruzzi, T. L., Fuller, T. K., Degraaf, R. M., Brooks, R. T. \& Li, W. 2002. Assessing remotely triggered cameras for surveying carnivore distribution. Wildlife Society Bulletin, 30: 380-386.

Petter, F. 1961. Répartition géographique et écologie des rongeurs désertiques de la région paléarctique. Mammalia, 25: 1-222.

Petter, F. 1987. Particularités écologiques et physiologiques des Rongeurs désertiques. Bulletin de l'Academie Veterinaire de France, 60: 159-163.

Petter, F., Lachiver, F. \& Chekir, R. 1984. Les adaptations des rongeurs Gerbillidés à la vie dans les régions arides. Bulletin de la Société Botanique de France. Actualités Botaniques, 131(2-4): 365-373.

Real, R., Guerrero, J. C., Márquez, A. L., Olivero, J. \& Vargas, J. M. 2003. Tipificación corológica de los micromamíferos ibéricos en relación con Europa y África. Graellsia, 59(2-3): 287-298. https://doi.org/10.3989/ graellsia.2003.v59.i2-3.247

Thomas, D. S. G. (ed.) 1997. Arid Zone Geomorphology: Process, Forms and Change in Drylands. John Wiley. Chichester. $713 \mathrm{pp}$.

UNEP (United Nations Environment Programme). 2004. Estratégie nationale pour la conservation et l'utilisation durable de la diversité biologique. L'Aménagement du territorie, de l'Eau et de l'Environnement du Maroc \& UNEP. Rabat. 181 pp.

Vilà, C., Amorim, I. R., Leonard, J. A., Posada, D., Castroviejo, J., Petrucci-Fonseca, F., Crandall, K., Ellegren, A., H. \& Wayne, R. K. 1999. Mitochondrial DNA phylogeography and population history of the grey wolf (Canis lupus). Molecular Ecology, 8(12): 2089-2103. https:// doi.org/10.1046/j.1365-294x.1999.00825.x

Wilson, I. G. 1972. Aeolian bedforms - their development and origins. Sedimentology, 19: 173-210. 